\title{
An Overview of QT Dispersion Finding in Cardiac Patients,Review Article Ahmed Mohamed Sanad ${ }^{1}$, Kamel Hasan Ghazal ${ }^{2}$, Mohammad Abdalla Eltahlawi ${ }^{2}$, and Ahmed Taha Abdelwahed ${ }^{2}$ \\ ${ }^{1}$ Kobri El-Kobba Military Hospital, Cairo, Egypt, ${ }^{2}$ Zagazig University, Zagazig, Egypt \\ *Corresponding Author: Ahmed Mohamed Sanad, E-Mail: dr_ahmed_sanad@yahoo.com
}

\begin{abstract}
Background: QT duration represents the time of the whole summated electric cardiac ventricular activity involving stepwise depolarization followed by repolarization. There has been a long history of using the surface electrocardiogram (ECG) to identify ventricular repolarization problems. The 1960s were a turning point for precise mathematical methodologies. It has been customary in clinical practice to use only the QT interval and the polarity and shape of the T wave when evaluating cardiac repolarization using an electrocardiogram (ECG). This terminology, such as "non-specific ST segment and T wave variations are widely used. An earlier theory on interlead disparities in QTI length was resurrected in a 1990 report by the group led by Professor John Campbell. The "QT dispersion" range of durations was proposed as a measure of ventricular recovery time spatial dispersion. Objective: Determine the relevance of QTd in prediction of myocardial and its severity. Conclusion: For cardiac patients, QTd is an easy-to-use, rapid, affordable, and helpful tool for helping with study interpretation, clinical management, and therapeutic orientation.
\end{abstract}

Keywords: QTd Dispersion Finding, Cardiac Patients

\section{QT dispersion Definition:}

The difference between 12-lead ECG's longest and shortest QT intervals can be described as QT Dispersion. These two extreme indices are calculated by measuring each one of 12 QT intervals separately ${ }^{(1)}$. This technique has a good correlation with the QT interval standard deviation across whole 12 leads. Instead of just two leads, the QT interval standard deviation could provide a more trustworthy dispersion measurement because it incorporates all of 12 lead values, lowering reliance on each lead's ability to detect the QT interval ${ }^{(2)}$.

\section{Long QT syndromes (LQTS):}

Despite the fact that most experts believe that LQTS patients have more QT dispersion, no connection was found between QT dispersion and symptoms according to Linker et al. ${ }^{(3)}$. Before and after beta blocker usage, QT dispersion did not differ significantly across the groups, according to this research. Priori et al. ${ }^{(4)}$, on the other hand, discovered that poor beta blocker treatment in LQTS patients was linked to persistently high QT dispersion values, as opposed to lower values found in individuals who reacted successfully by use of this medication. QTc dispersion was found to decrease both before and during beta blockage in normal subjects but increased abnormally in LQTS subjects due to exercise. This abnormal rise was avoided by beta blockers usage. Beta blocking medications have been shown to protect against LQTS arrhythmias, which are usually caused by stress, according to the authors (5).

After beta blockers were used in LQT1 group, the mean $T_{p e}$ values were no longer prolonged in the same way as they were in the control group without beta blockers utilization. In LQT1 patients, our findings show that the use of BBs reduces the risk of ventricular repolarization arrhythmia. Sudden cardiac arrest and syncope risks can be reduced in this population by BBs taking ${ }^{(6)}$.

\section{Pathophysiology of QT dispersion:}

Increased QTd has a complex pathophysiology that is still being studied. Regional changes in ventricular wall stress (contraction-excitation feedback or mechanoelectric) generated by ventricular dilatation and fibrosis may increase the refractoriness' dispersion. This theory is backed up by research showing elevated QTd in patients with acquired long QT interval, such as those with hypertension, hypertrophic cardiomyopathy, left ventricular hypertrophy, and acute $\mathrm{MI}^{(7)}$.

On top of that, it's unclear what causes the QTd seen over surface of the body to appear so long. Because action potential duration and activation time vary regionally, which causes repolarization heterogeneity. Repolarization dispersion can be caused by changes in the action potential duration between cell groups coming from different levels of the ventricular myocardium, as evidenced by research in this subject. In comparison to endocardial or epicardial cell layers, the presence of M-cells (in the mid-myocardial layer) has been shown to be accompanied by repolarization prolongation $^{(\mathbf{1})}$.

It was discovered by Gokhan et al. (8) that prolongation of QT over ECG indicates a non-uniform and delayed repolarization recovery in regions of infarction or ischemia. Ischemia elevates potassium level extracellularly, causing anoxia and acidosis, these are the fundamental mechanisms. This also reduces membrane excitability, shortens action potentials, and extends the time it takes for excitability to recover once an action potential has occurred. Myocardial no-reflow syndrome is thought to be caused in part by embolization of thrombotic material.

The epicardiac layer's action potential ended at the Twave peak in the body surface ECG, while the midmyocardial layer's repolarization ended at the T-wave offset (containing M-cells). T-wave peak (Tp) to T-wave end $(\mathrm{Te})$ time difference signifies the repolarization transmural dispersion from this perspective ${ }^{(9)}$. 
This means that the ventricular recovery dispersion duration evaluated with by QT dispersion and monophasic action potentials (MAPs) is a and indirect and direct repolarization representation alterations that has a high probability of being connected even if there isn't a mechanism to tie everything together. The spatial $\mathrm{T}$ wave loop is altered by ventricular repolarization anomalies that are not limited to those that cause regional dispersion in recovery times. A change among normality of loop projections into various leads of ECG could make finding ECG tracings more difficult. QT dispersion can be explained by looking at the influence local repolarization dispersion has on $\mathrm{T}$ wave shape. The real mechanical background of In the T wave loop dynamics, QT dispersion appears to be a variable projection of the loop into distinct ECG leads (10).

Further local electro-physiological inhomogeneity can be generated by a differential contraction-excitation feedback when there are regional wall motion alterations. There is evidence to suggest that acute myocardial infarction with Q wave have greater QT dispersion, and this may be due to the enhanced dilatation and remodeling seen in this group of patients. After a myocardial infarction, neurohormonal systems are always activated, and this is especially true in left ventricular dysfunction patients. Acting on myocytes with varying levels of viability and electrophysiological qualities could exacerbate the difference already present in ventricular repolarization patterns. In addition, excessive angiotensin II levels and patchy cardiac fibrosis might cause this ${ }^{(\mathbf{1 1})}$.

\section{QT dispersion Measurements:}

All ECG 12 leads must be captured concurrently for prevention of the effect of QT dynamicity caused by changes in heart rate for an accurate assessment of QT dispersion. A "gold standard" has been established for measuring QTd using 12-lead recordings made simultaneously. But on the other hand, because of the QT interval's sluggish dynamic nature, QT dispersion measures based on six or three simultaneous recordings during an ectopic-free sinus rhythm may be satisfactory in practice. However, there is no solid evidence to support this strategy (12).

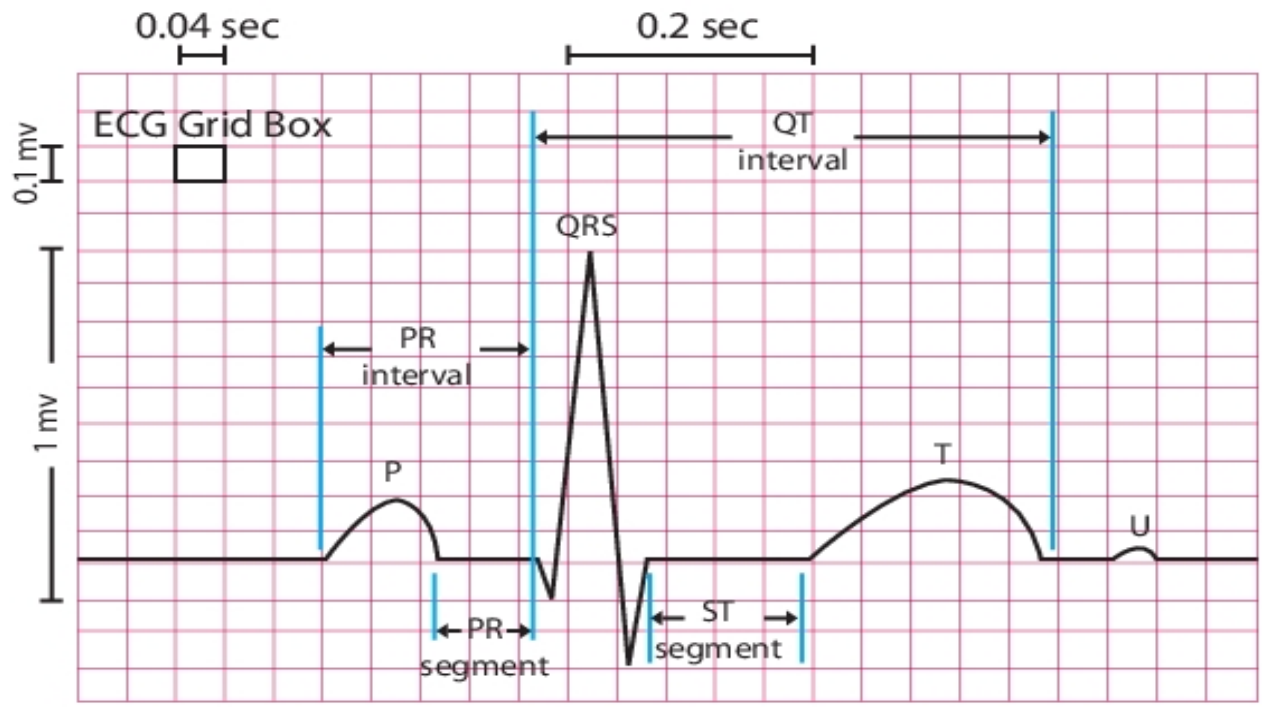

Fig. (1): ECG determination of QT interval ${ }^{(\mathbf{1})}$.

The difference between the longest (QT max) and the shortest (QT min) mean QT intervals in each ECG was used to compute QT dispersion (QTd) in milliseconds. QTd is equal to (QTmax- $(\mathrm{QTmin})^{\mathbf{( 1 )}}$. QTd $=30-60 \mathrm{~ms}$ was considered normal (13).

The QT interval is calculated by comparing the beginning of the QRS complex with the end of the T wave, and then applying the formula below: (A) the descending portion of the $\mathrm{T}$ wave returns to the isoelectric baseline at which it terminates. (B) The reference line separating two PQ intervals serves as the isoelectric baseline. (C) When a U wave is present, the nadir point between the $\mathrm{T}$ and $\mathrm{U}$ waves is considered the end of the $\mathrm{T}$ wave. (D) Leads were discarded from the study if the $\mathrm{T}$ wave could not be accurately measured (for amplitudes less than $50 \mu \mathrm{V}$ ) ${ }^{(\mathbf{1 4})}$.

Manually determining the $\mathrm{T}$ wave offset has long been recognized to be not reliable. There is no evidence to suggest that currently available automated procedures are better than manual approaches. Low $\mathrm{T}$ wave amplitude and $\mathrm{T}$ wave merging with $\mathrm{U}$ or $\mathrm{P}$ waves are the primary sources of mistake for human observers as well as computers. The form of the $\mathrm{T}$ wave has a significant impact on the measurement of the QT interval. There are a number of simple algorithms for determining the end of the T wave automatically ${ }^{(15)}$. 

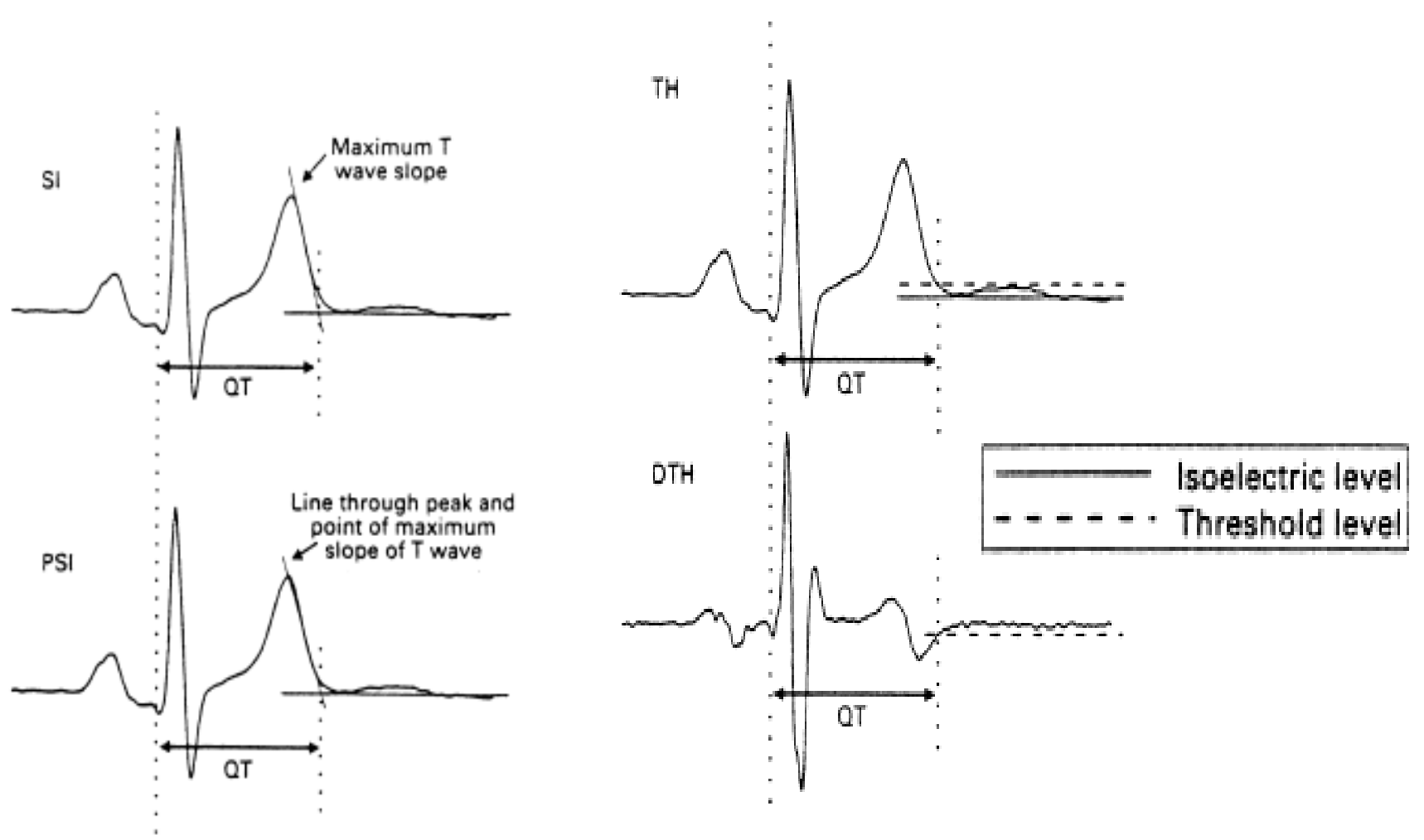

Fig. (2) : Techniques for QT automatic measurement ${ }^{(\mathbf{1 5})}$.

$\mathrm{T}$ wave threshold approach, tangent technique utilizing the steepest point of the $\mathrm{T}$ wave limb descent (SI), and differential threshold approach (DTH): a line drawn from the apex of the $\mathrm{T}$ wave to the highest slope point using the tangent technique (PSI). This approach finds the $\mathrm{T}$ offset as a point where a slope-to-isoelectric-line intercept occurs, or as a threshold line above the $\mathrm{T}$ wave's declining portion. Depending on the line fitting technique, the steepest tangent or a connection between inflex point to the $T$ wave's apex as straight line might be used as the slope. The recorded QT interval values are clearly influenced by the descending $\mathrm{T}$ wave's form. In both automatic and manual measurements, the amplitude of the $\mathrm{T}$ wave has a significant impact on measurement dependability ${ }^{\left({ }^{16}\right)}$.

\section{1-Increased QT dispersion in LVH: possible Mechanisms:}

At the microscopic level, LVH shows signs of increase in interstitial matrix collagen and hypertrophied myocytes. Electrical quiescence along with an increase in myocyte hypertrophy may occur with interstitial fibrosis in addition to a decrease in the amplitude and membrane potential of the action potentials or a reduction in the duration of the action potentials. If the modifications in the ventricle are not homogeneous, each of these aspects could lead to increased QT dispersion, and it is obvious how such changes can lead to ventricular arrhythmias and reentry circuits and ventricular arrhythmias ${ }^{(17)}$.

\section{2-QT dispersion and Non-STEMI:}

QT dispersion can indicate successful reperfusion in patients with non-ST-segment acute myocardial infarction.
Reduction of QTd on ECG surface before as well as after PCI to restore epicardial blood flow can serve as a sign of effective reperfusion therapy in individuals with acute nonSTEMI. To better detect reperfusion in individuals with non-transmural infarction, a 50 percent drop in QTd cutoff value has been proposed ${ }^{(18)}$.

\section{3-QT dispersion and incidence of arrhythmia:}

Regional differences in cardiac recovery of excitability have been linked to higher QT dispersion on the ECG ${ }^{(\mathbf{1 9})}$. Consequently, a more arrhythmogenic substrate may be created. Individuals with long QT syndrome with a high risk of sudden death have an elevated risk of arrhythmias when their QT dispersion is raised ${ }^{\mathbf{2 0})}$.

\section{4-Other Diseases:}

Patients with peripheral vascular disease who do not have overt ischemic heart disease have a QT dispersion of higher than $60 \mathrm{~ms}$, which predicts cardiac death $(81 \%$ specificity, $92 \%$ sensitivity). Those with a long QT interval (>120 ms) on the heart transplant waiting list had a fourfold increase in mortality that occurs prior to transplant operation ${ }^{(21)}$.

Heart valve prolapse patients had longer QT intervals, which may help explain why ventricular dysrhythmias are so common in this group of people ${ }^{(22)}$. Rheumatoid arthritis and systemic sclerosis patients have both been found to have prolonged QTc dispersion and QT ${ }^{(23)}$.

However, despite the drawbacks, the decade-long study of QT dispersion highlighted the clinical significance of repolarization anomalies Our understanding of QT dispersion must include the understanding that it is nothing 
more (or less) than an approximate and rudimentary depiction of repolarization irregularity.

\section{Measurements features:}

A technical investigation by Malik and Bradford ${ }^{(10)}$ shows that the gold standard technique using digitizing board for manual measurement could have differences due to totally error-related QT dispersion, while $40 \mathrm{~ms}$ and 60 $\mathrm{ms}$, respectively, were recorded by $20 \%$ and $10 \%$ of observers. No better consistency has been demonstrated for the currently available automatic QT measuring methods.

The instantaneous reliability of various QT dispersion indices calculated with a downslope tangent approach in healthy volunteers, for example, was reported by Yi $\boldsymbol{e t}$ al. (24) to range between 16 and 44 percent (in sequentially recorded ECGs). The way the tangent is characterized, the definition of the isoelectric line, the threshold level, and so on are all examples of computer algorithm variations for determining $\mathrm{T}$ wave offset.

\section{Heart rate:}

The so-called corrected QT dispersion, i.e., the QT interval dispersion corrected for heart rate by some formula, was utilized in many investigations, including major prospective evaluations. The dispersion of ventricular recovery times can be influenced by the rate, rhythmicity, and origin of the impulse; however, this has never been demonstrated for QT dispersion ${ }^{(25)}$.

Heart rate had no link with ventricular recovery time dispersion, as determined by MAPs or QT dispersion in any of the investigations. The relationship between recovery time dispersion and heart rate is still a debate. The QT dispersion in a typical 12-lead ECG, on the other hand, is clearly not dependent on the cardiac period in the same way that the QT interval is (that's why it should not be reliable) (26).

As opposed to previous cardiac cycle duration, the QT interval duration is dependent on underlying heart rate (27), Although the QT interval length is determined by the underlying heart rate rather than the cardiac cycle's duration. Bazette formula which was described in 1920 $(\mathrm{QTc}=\mathrm{QT} / \sqrt{\mathrm{RR}})$ is the most widely used for correction of QT to heart rate. This formula was refused by some studies as it causes over-correction if heart rate above 100 beats/min (28). Other QTc formulae, e.g. Fridericia, Framingham, and Hodges, are also used.

\section{Role of ECG system and number of Leads:}

QT interval durations are influenced by the conventional ECG's number of detectable leads. The socalled adjusted QT dispersion was developed by certain studies, who divided the QT interval range by the square root of the number of detectable ECG leads to get at the adjustment factor ${ }^{(20)}$. Others have demonstrated that using this approach correctly corrects the mean QT dispersion values found in normal ECGs. However, they also demonstrated that missing individual leads causes large numbers of little errors. It is therefore inappropriate to compare QT interval values recorded in ECGs with substantially varied numbers of measurable leads in order to draw any conclusions ${ }^{(2)}$.

\section{CONCLUSION}

For cardiac patients, QTd is an easy-to-use, rapid, affordable, and helpful tool for helping with study interpretation, clinical management, and therapeutic orientation.

\section{REFERENCES}

1. Sahu P, Lim P, Rana B et al. (2000): QT dispersion in medicine: electro-physiological Holy Grail or fool's gold. QJM. 93(7):425-31.

2. Hnatkova K, Malik M, Kautzner J et al. (1994): Adjustment of QT dispersion assessed from 12 lead electrocardiograms for different numbers of analyzed electrocardiographic leads: comparison of stability of different methods. Br Heart J., 72(4):390-6.

3. Linker $\mathrm{N}$, Colonna $\mathrm{P}$, Kekwick $\mathrm{C}$ et al. (1992): Assessment of QT dispersion in symptomatic patients with congenital long QT syndromes. Am J Cardiol., 69(6):6348.

4. Priori S, Napolitano C, Schwartz P et al. (2004): Association of long QT syndrome loci and cardiac events among patients treated with beta-blockers. JAMA. 292(11):1341-4.

5. Fu G, Meissner A, Simon R (1997): Repolarization dispersion and sudden cardiac death in patients with impaired left ventricular function. Eur Heart J., 18(2):2819.

6. Lee W, Gregory M, Mary M et al. (2011): Beta-blockers protect against dispersion of repolarization during exercise in congenital long-QT syndrome type 1. J Cardiovasc Electrophysiol., 22(10): 1141-1146.

7. Clarkson $P$, Naas A, McMahon A et al. (1995): QT dispersion in essential hypertension. Q J Med., 8:327-332.

8. Gokhan A, Muslum S, Birol $\mathrm{O}$ et al. (2013): The Comparison in Reduction of QT Dispersion After Primary Percutaneous Coronary Intervention According to Existence of Thrombectomy in STEMI. Clin Cardiol., 36(5): 276-279.

9. Antzelevitch C, Shimizu W (1998): Cellular basis for the ECG features of the LQT1 form of the long-QT syndrome: effects of beta-adrenergic agonists and antagonists and sodium channel blockers on transmural dispersion of repolarization and torsade de pointes. Circulation, 98(21):2314-22.

10. Malik M, Batchvarov V (2000): Measurements and interpretation of QT dispersion. Progress Cardiovasc Dis., 42:325-344.

11. Taggart P, Sutton $P$, John $R$ et al. (1992): Monophasic action potential recordings during acute changes in ventricular loading induced by the valsalva manoeuver. $\mathrm{Br}$ Heart J., 67:221-229.

12. Lau C, Freedman A, Fleming S et al. (1987): Hysteresis of the ventricular paced QT interval in response to abrupt changes in pacing rate. Cardiovasc Res., 22:67-72.

13. Mirvis D, Goldberger A (2012): Electrocardiography. In: Bonov RO, Mann DL, Zipes DB, Libby P, editors. Braunwald's Heart Disease: A Textbook of Cardiovascular Medicine. 9th edn. Elsevier Sanders; Pp. 126-167.

14. Zimarino M, Corazzini A, Tatasciore A et al. (2011): Defective recovery of QT dispersion predicts late cardiac 
mortality after percutaneous coronary intervention. Heart, 97(6):466-472.

15. McLaughlin N, Campbell R, Murray A (1995): Comparison of automatic QT measurement techniques in the normal 12 lead electrocardiogram. Br Heart J., 74:8489.

16. Kors J, Van Herpen G (1998): Measurement error as a source of QT dispersion: a computerized analysis. Heart, 80(5):453-8.

17. Pye M, Cobbe S (1992): Mechanisms of ventricular arrhythmias in cardiac failure and hypertrophy. Cardiovasc Res., 26(8):740-750.

18. Jensen C, Lusebrink $S$, Wolf A et al. (2015): Reduction of QTD - A novel marker of successful reperfusion in NSTEMI. Pathophysiologic Insights by CMR. Int J Med Sci., 12(5): 378-386.

19. Higham P, Furniss S, Campbell R (1995): QT dispersion and components of the QT interval in ischemia and infarction. Br Heart J., 73(1):32-36.

20. Day C, McComb J, Campbell R (1990): QT dispersion: an indication of arrhythmia risk in patients with long QT intervals. Br Heart J., 63(6):342-344.

21. Pinsky D, Sciacca R, Steinberg S (1998): QT dispersion as a marker of risk in patients awaiting heart transplantation. J Am Coll Cardiol., 29(7):1576-1584.

22. Vloka M, Babaev A, Ehlert F et al. (1999): Poor correlation of automated and manual QT dispersion measurements in patients and normal subjects (abstr). J Am Coll Cardiol., (33): 350-55.

23. Goldeli O, Dursun E, Komsuoglu B (1998): Dispersion of ventricular repolarization; a new marker of ventricular arrhythmias in patients with rheumatoid arthritis. J Rheumatol., 25:447-450.

24. Yi G, Guo X, Crook R et al. (1998): Computerized measurements of QT dispersion in healthy subjects. Heart, 80:459- 66 .

25. Okin P, Devereux R, Jern S et al. (2000): Baseline characteristics in relation to electrocardiographic left ventricular hypertrophy in hypertensive patients: the Losartan Intervention for Endpoint reduction (LIFE) in hypertension study. The Life Study Investigators. Hypertension, 36(5):766-773.

26. Zabel M, Hohnloser S (1997): Pathophysiologische Bedeutung und prognostische Wertigkeit der QT dispersion im Oberflachen-EKG. Elektrophys, 8:223-226.

27. Franz M, Swerdlow C, Liem L et al. (1998): Cycle length dependence of human action potential duration in vivo. Effects of single extrastimuli, sudden sustained rate acceleration and deceleration, and different steady-state frequencies. J Clin Invest., 82: 972-979.

28. Davey P, Bateman J (1999): Heart rate and catecholamine contribution to QT interval shortening on exercise. Clin Cardiol., 22:513-518. 